http://dx.doi.org/10.22319/rmcp.v10i1.4451

Nota de investigación

\title{
Composición botánica y valor nutritivo de la dieta consumida por bovinos en un área invadida por pasto rosado [Melinis repens (willd.) Zizka]
}

Obed Gabriel Gutiérrez Gutiérrez ${ }^{\mathrm{a}}$

Carlos Raúl Morales Nieto ${ }^{\text {a* }}$

José Carlos Villalobos González ${ }^{\mathrm{b}}$

Oscar Ruíz Barrera ${ }^{\mathrm{a}}$

Juan Ángel Ortega Gutiérrez ${ }^{\mathrm{a}}$

Jorge Palacio Nuñez ${ }^{\mathrm{c}}$

${ }^{a}$ Universidad Autónoma de Chihuahua. Facultad de Zootecnia y Ecología. Km 1 Perif. Francisco R. Aldama, 31453, Chihuahua, Chih. México.

b Texas Tech University. Lubbock Texas. USA.

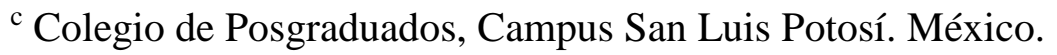

*Autor para correspondencia: cnieto@uach.mx

\section{Resumen:}

Se evaluó la composición botánica y el valor nutricional de la dieta de ganado bovino en áreas invadidas por pasto rosado [Melinis repens (Willd.) Zizka]. La investigación se realizó en el Rancho Salinas, municipio de Satevó, Chihuahua, en un pastizal amacollado arbosufrutescente. La composición botánica del área se determinó por el método de línea de puntos. Los muestreos se realizaron de agosto de 2013 a febrero de 2014 y se utilizaron dos vaquillas fistuladas del esófago de la cruza Hereford-Angus de $350 \pm 5 \mathrm{~kg}$. La composición botánica de la dieta se obtuvo mediante la técnica microhistológica y su valor nutricional por análisis bromatológico. Los datos se sometieron a un análisis de varianza; los datos de calidad de la dieta se ajustaron a un modelo mixto mediante el procedimiento PROC MIXED del SAS. El promedio de forraje disponible durante las cuatro etapas fenológicas fue de 1,279 $\mathrm{kg}$ MS ha-1, con presencia del pasto rosado del $87.5 \%\left(1,119.13 \mathrm{~kg} \mathrm{MS} \mathrm{ha}{ }^{-1}\right)$. La dieta seleccionada por el ganado bovino estuvo compuesta por $74 \%$ de gramíneas, $19 \%$ de 
arbustivas y $7 \%$ de herbáceas. Las especies preferidas fueron Aristida divaricata (8.43) y Croton pottsii (12.95) durante la etapa de crecimiento; el menor índice de preferencia fue para Melinis repens $(0.33$ a 0.41$)$. El mayor contenido de proteína cruda se obtuvo durante las etapas de crecimiento (13.23\%) y floración (10.71\%). La mejor calidad de la dieta se presentó durante las etapas de crecimiento y floración, y estuvo compuesta principalmente por Melinis repens en las cuatro etapas.

Palabras clave: Índice de preferencia, Consumo voluntario, Melinis repens.

Recibido: 25/03/2017

Aceptado: 06/03/2018

La producción de carne en los sistemas extensivos depende principalmente de la cantidad y calidad de los pastos presentes en los agostaderos y representan el alimento más barato para el ganado. En el norte de México las precipitaciones se presentan por lo regular durante los meses de junio a septiembre $(80 \%)$, esto provoca que las mayores ganancias de peso y rentabilidad del sistema ocurran durante esta época ${ }^{(1)}$.

Una de las actividades económicas más importantes para los ganaderos en el estado de Chihuahua es el sistema vaca-cría, el cual consiste en la producción de becerros para exportación $^{(2,3)}$. Sin embargo, en los últimos años la ganadería ha sufrido pérdidas por sequía y mal manejo del pastizal; el resultado ha sido una pérdida en la producción del pastizal y una disminución del hato ganadero; esto debido a ventas forzadas y sacrificio del ganado en mala condición ${ }^{(4,5)}$. Además, extensas áreas del pastizal han sufrido cambios en su estructura debido a la introducción e invasión de especies no nativas, las cuales están provocando un impacto ecológico por la pérdida de especies forrajeras nativas ${ }^{(6,7)}$.

El pasto rosado (Melinis repens) es una especie oportunista que se ha extendido en los últimos 30 años a lo largo del territorio mexicano ${ }^{(7)}$, ya que se establece rápidamente y desplaza especies nativas de importancia ganadera, ecológica y económica ${ }^{(8)}$. Su valor forrajero va de regular a malo durante la época de latencia, donde puede alcanzar valores de proteína cruda (PC) del $4 \%^{(7)}$. No obstante, se desconoce el grado de preferencia por los animales en pastoreo. En Chihuahua, esta especie se ha desplazado del centro-sur hacia el norte del Estado y se localiza principalmente en lugares que han sido degradados por el sobrepastoreo y en tierras abandonadas que fueron abiertas al cultivo.

Los estudios de dietas de bovinos en pastoreo realizados en Chihuahua, datan de la década de los años 80s; dichos estudios se ubicaron en áreas de la región centro-norte y en épocas donde los procesos de invasión del $M$. repens estaban en una etapa incipiente o aún no se encontraba presente ${ }^{(9,10,11)}$. El conocimiento de la composición de la dieta, así como el aporte 
nutricional de las plantas forrajeras en áreas invadidas, puede ayudar a diseñar y ejecutar esquemas de uso y manejo, tanto del pastizal como de los animales en pastoreo.

Diversas técnicas se han utilizado para conocer la composición botánica de la dieta de ganado bovino en pastoreo. Una de estas técnicas es la microhistológica, que permite identificar y cuantificar la composición botánica de la dieta consumida ${ }^{(11,12)}$. Además, el conocimiento del valor nutricional de la dieta del ganado bovino en pastoreo, es importante para establecer la suplementación necesaria durante la época crítica, que no cubre los requerimientos de los animales. Por lo anterior, y debido a que se carece de información sobre pastizales invadidos con $M$. repens $^{(13)}$, se planteó la presente investigación cuyo objetivo fue evaluar la composición botánica y valor nutricional en la dieta de bovinos en pastoreo en un área invadida por $M$. repens.

La presente investigación se realizó en el Rancho "Salinas", ubicado en el municipio de Satevó, Chihuahua, localizada a $27^{\circ} 57^{\prime} 00^{\prime}$ ' N y $106^{\circ} 07^{\prime} 00$ ”' O y 1,540 msnm. Temperatura media anual de $18.1^{\circ} \mathrm{C}$ y precipitación promedio anual histórica de $464 \mathrm{~mm}^{(14)}$. Sin embargo, en el año 2013 de acuerdo a CONAGUA ${ }^{(15)}$ hubo una precipitación promedio de $550 \mathrm{~mm}$. La precipitación durante el año de estudio se distribuyó principalmente de julio a septiembre y noviembre (Figura 1). Su clasificación climática según Köppen es BWh ${ }^{(16)}$. Los suelos son someros (0 a $25 \mathrm{~cm})$, drenaje interno regular y $\mathrm{pH}$ de 5.3 a $6.6^{(16)}$.

Figura 1: Precipitación $(\mathrm{mm})$ promedio en el año 2013 durante el período de estudio ${ }^{(14)}$

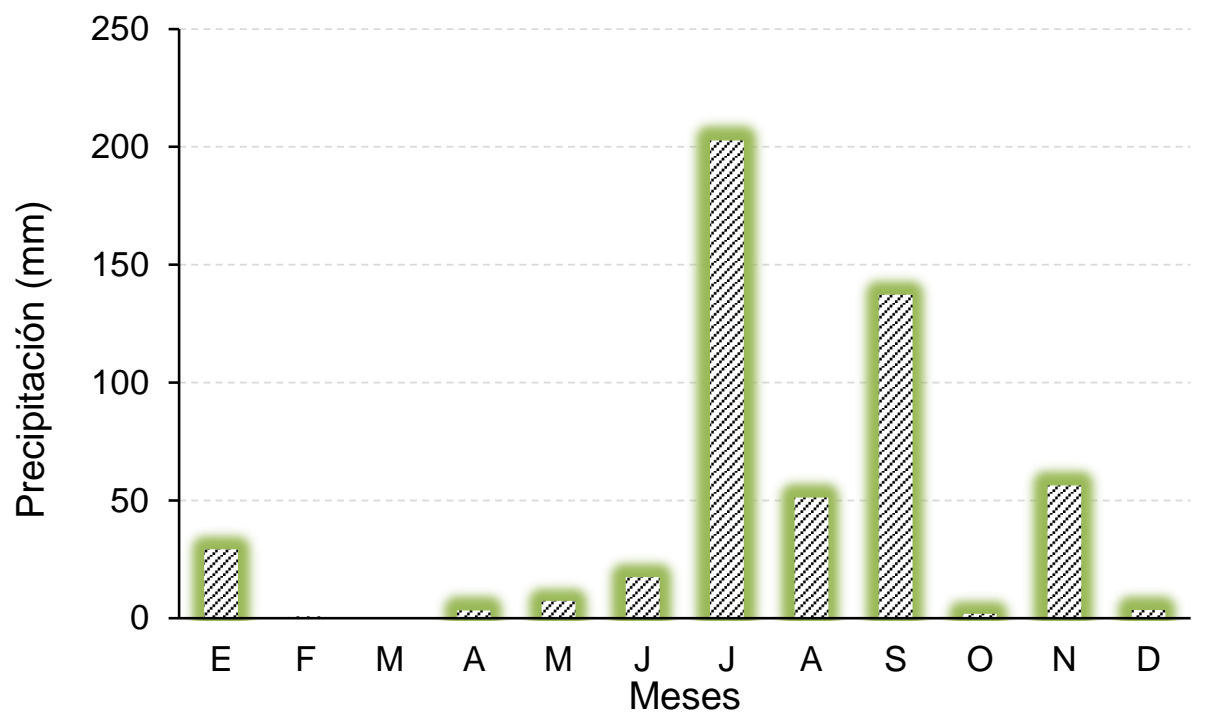

El área de estudio tiene una extensión de 200 ha con un coeficiente de agostadero de 4 ha $\mathrm{UA}^{-1}$, el cual se determinó con base en la cantidad de forraje utilizable $\left(1,470 \mathrm{~kg} \mathrm{ha}^{-1}\right)$ y los requerimientos del tipo de animal ${ }^{(10,17)}$. Esta área se seleccionó por ser representativa de los pastizales invadidos con $M$. repens. El tipo de vegetación es un pastizal amacollado 
arborescente con invasión de $M$. repens y presencia de especies arbustivas como Mimosa biuncifera, Prosopis glandulosa y gramíneas como Bouteloua gracilis, Bouteloua curtipendula, entre otras (Cuadro 1) ${ }^{(16,18)}$.

Cuadro 1: Composición botánica general en el área de estudio durante la etapa de crecimiento

\begin{tabular}{|c|c|c|c|c|c|}
\hline Gramíneas & $\begin{array}{c}\text { Cobertura } \\
(\%)^{1}\end{array}$ & Arbustivas & $\begin{array}{c}\text { Cobertura } \\
(\%)^{1}\end{array}$ & Herbáceas & $\begin{array}{c}\text { Cobertura } \\
(\%)^{1}\end{array}$ \\
\hline $\begin{array}{l}\text { Bouteluoa } \\
\text { curtipendula }\end{array}$ & 0.41 & Aloysia wrightii & 1.46 & $\begin{array}{l}\text { Bulbostilis } \\
\text { juncoides }\end{array}$ & 0.63 \\
\hline Bouteluoa gracilis & 2.50 & $\begin{array}{l}\text { Calliandra } \\
\text { eriophylla }\end{array}$ & 9.60 & $\begin{array}{c}\text { Dichondria } \\
\text { argentea }\end{array}$ & 0.41 \\
\hline \multirow[t]{7}{*}{ Melinis repens } & 63.72 & Condalia sp. & 0.21 & Euphorbia sp. & 0.21 \\
\hline & & $\begin{array}{c}\text { Juniperus } \\
\text { monosperma }\end{array}$ & 1.25 & $\begin{array}{l}\text { Evolvolus } \\
\text { alsynoides }\end{array}$ & 9.60 \\
\hline & & Mimosa biuncifera & 1.67 & $\begin{array}{l}\text { Haploppapues } \\
\text { gracilis }\end{array}$ & 0.21 \\
\hline & & $\begin{array}{l}\text { Prosponis } \\
\text { glandulosa }\end{array}$ & 2.50 & $\begin{array}{l}\text { Macrosiphonia } \\
\text { hypoleuca }\end{array}$ & 0.21 \\
\hline & & Tecoma stands & 0.21 & Millia biflora & 0.62 \\
\hline & & & & Sida procumbens & 3.76 \\
\hline & & & & Croton pottsii & 1.00 \\
\hline
\end{tabular}

${ }^{1}$ Determinada mediante el método de puntos de intercepción ${ }^{(18)}$.

Para determinar tanto la composición botánica de la dieta como la calidad de la misma, se realizaron cuatro muestreos (agosto de 2013 a febrero de 2014), correspondientes a las etapas fenológicas de las gramíneas (crecimiento, floración, madurez y latencia) presentes en el área (Cuadro 2). El primer muestreo se realizó durante la etapa de crecimiento (3 al 7 de agosto); el segundo durante la etapa de floración (1 al 5 de octubre); en la etapa de madurez el muestreo se realizó del 3 al 7 de diciembre; y el muestreo de la etapa de latencia se realizó del 1 al 5 de febrero del 2014. Para estos muestreos se utilizaron dos vaquillas con peso promedio de $350 \pm 5 \mathrm{~kg}$ de la cruza Hereford y Angus, fistuladas (fístula marca Bar Diamond) del esófago ${ }^{(19,20,21)}$. Holechek ${ }^{(12)}$ menciona que en pastizales donde la vegetación es muy homogénea se puede obtener la composición botánica con dos animales durante dos a tres días; debido a lo anterior, se utilizaron dos animales fistulados y se dio un periodo de pastoreo de 5 días. 
Cuadro 2: Composición botánica (\%) de gramíneas presentes en el área de estudio

\begin{tabular}{lcccc}
\hline \multirow{2}{*}{ Especies } & \multicolumn{4}{c}{ Etapas de muestreo } \\
\cline { 2 - 5 } & Crecimiento & Floración & Madurez & Latencia \\
\hline Aristida divaricata & $0.57^{1}$ & $--^{2}$ & $-{ }^{2}$ & $0.61^{1}$ \\
Bothriochloa barbinodis & -- & 4.12 & 1.15 & 0.81 \\
Bouteloua chondrosioides & -- & 4.67 & 0.37 & 0.99 \\
Bouteloua curtipendula & 9.58 & 7.61 & 3.26 & 3.60 \\
Bouteloua gracilis & 10.15 & 7.35 & 4.89 & 3.49 \\
Bouteloua hirsuta & -- & 2.44 & 1.75 & 1.32 \\
Heteropogon contortus & -- & -- & 1.01 & 1.48 \\
Leptochloa dubia & -- & 1.11 & -- & 0.90 \\
Muhlenbergia phleoides & -- & 1.01 & 0.61 & 0.86 \\
Melinis repens & 79.68 & 71.68 & 86.96 & 85.94 \\
\hline
\end{tabular}

${ }^{1}$ Valores calculados con base en la producción de biomasa.

${ }^{2}$ No observado en la lectura.

A los animales se les dio un periodo de adaptación de tres días en el área de estudio antes de cada muestreo. Los animales se dietaron por la noche para evitar la contaminación de muestras. Estos fueron colocados durante la mañana en el área de estudio durante 45 a 60 min; transcurrido este periodo las bolsas fueron retiradas con la muestra recolectada. Las muestras se trasladaron al laboratorio de nutrición animal de la Facultad de Zootecnia y Ecología; para su secado se colocaron en una estufa de aire forzado a $60{ }^{\circ} \mathrm{C}$ por $72 \mathrm{~h}$, y se molieron en un molino Wiley® con malla de $1 \mathrm{~mm}$ (Arthur H. Tomas, Philadelphia, PA, USA) $)^{(22,23)}$.

Rendimiento de materia seca. Para estimar el rendimiento de materia seca ( $\left.\mathrm{kg} \mathrm{MS} \mathrm{ha}{ }^{-1}\right)$ se seleccionaron al azar 25 puntos y se utilizó un cuadrante de $0.25 \mathrm{~m}^{2}$ para realizar los cortes de forraje. Las gramíneas (se separaron por especie) que se encontraban dentro de cada cuadrante se cortaron a ras del suelo. Las muestras de forraje se colocaron en una estufa a $75^{\circ} \mathrm{C}$ durante $72 \mathrm{~h}$ para su secado y posterior pesaje en el Laboratorio de Nutrición Animal de la FZyE-UACH. Con los pesos obtenidos se calculó el porcentaje de cada especie, durante las cuatro etapas fenológicas. Para el pesaje se utilizó una báscula marca Tor Rey L-EQ y el peso seco obtenido se extrapoló a producción de MS por hectárea, utilizando la siguiente fórmula:

$$
\mathrm{MS} \mathrm{ha}^{-1}=\frac{\text { peso de la muestra seca }(\mathrm{g}) * 4}{1000 \mathrm{~g}} * 10,000
$$


Composición botánica del área. La composición botánica se midió de dos maneras: en el primer caso se realizó un estudio general de la vegetación presente en el mes de julio mediante la técnica de líneas de puntos $^{(18)}$, con la finalidad de tener una referencia de las especies presentes en el área de estudio. Lo anterior, debido a que las arbustivas no cambian su composición durante las diferentes etapas, por lo cual, solo se midieron durante el mes de julio $^{(17)}$. En el segundo caso se midió la presencia y ausencia de las gramíneas durante cada fecha de muestreo, con la ayuda de un cuadrante de $0.25 \mathrm{~m}^{2}$.

Composición botánica de la dieta. La identificación de los tejidos vegetales de las especies presentes en la dieta del ganado, se realizó mediante el uso de la técnica microhistológica modificada por Peña y $\mathrm{Habib}^{(11)}$; la cual considera la lectura de cinco laminillas y 20 campos en cada una de éstas. El trabajo se desarrolló en el laboratorio de Agua-Suelo-Planta del Colegio de Posgraduados (COLPOS), Campus SLP.

Índice de preferencia. Para los animales en pastoreo, el índice de preferencia de las especies presentes en el área de estudio, se estimó mediante la composición botánica de la dieta y la composición botánica del área y se utilizó la fórmula propuesta por Van Dyne y Heady ${ }^{(24)}$.

$$
\mathrm{IP}=\frac{\text { Composición botánica de la dieta }}{\text { Composición botánica del área }}
$$

Los valores altos significan mayor preferencia por el ganado y valores pequeños significa menor preferencia.

Composición química de la dieta. Las muestras recolectadas se colocaron en una estufa para su secado y posterior molido. Después de la preparación de las muestras (30 por cada periodo de muestreo), se realizaron análisis químicos para determinar proteína cruda y digestibilidad in vitro. Estos se realizaron en el laboratorio de nutrición animal de la FZyE-UACH. El contenido de proteína cruda (PC) se determinó por el método Kjeldahl: la cantidad de nitrógeno $(\mathrm{N})$ en la muestra se multiplica por el factor $6.25^{(22)}$. Además, la materia orgánica digestible in vitro se determinó en el analizador Daysi II, siguiendo la metodología propuesta por Ankom ${ }^{\circledR}$ (Ankom Technology, Fairport, NY, USA) ${ }^{(23)}$. Para las concentraciones de fibra detergente neutro (FDN) y fibra detergente ácido (FDA) se utilizó la metodología propuesta por Van Soest et $a l^{(25)}$.

Para realizar el análisis estadístico de la composición botánica de la dieta, se incluyó únicamente la categoría: gramíneas, herbáceas y arbustivas. Los datos se sometieron a un análisis de varianza en un diseño completamente al azar $(\alpha=0.05)$, utilizando el procedimiento MIXED del programa $\mathrm{SAS}^{(26)}$. La ecuación del modelo estadístico utilizado fue:

$$
y_{i j}=\mu+P_{i}+e_{i j}
$$




\section{Donde:}

$\boldsymbol{y}_{\boldsymbol{i j}}=$ valor de la variable respuesta (cada especie y las agrupaciones) observado en el $i$-ésimo estado fenológico;

$\mu=$ efecto de la media general;

$\boldsymbol{P}_{\boldsymbol{i}}=$ efecto fijo del $i$ ésimo estado fenológico;

$\boldsymbol{e}_{i j}=$ término del error aleatorio asociado a las observaciones, donde $e_{i j} \sim N I I D\left(0, \sigma^{2}\right)$.

Los datos de la composición química se analizaron por varianza, ajustando un modelo mixto mediante el procedimiento PROC MIXED del SAS ${ }^{(26)}$, con la ecuación:

$$
y_{i j k}=\mu+P_{i}+V_{j}+\theta_{i j}+e_{i j k}
$$

\section{Donde:}

$\boldsymbol{y}_{\boldsymbol{i} \boldsymbol{j} \boldsymbol{k}}=$ valor de la variable respuesta (MO, PC, DivMO, FDN, FDA) observado en el $i$-ésimo estado fenológico en el $j$-ésimo animal fistulado;

$\mu=$ efecto de la media general;

$\boldsymbol{P}_{i}=$ efecto fijo del $i$ ésimo estado fenológico;

$\boldsymbol{V}_{\boldsymbol{j}}=$ efecto aleatorio del $j$ ésimo animal fistulado;

$\boldsymbol{\theta}_{\boldsymbol{i} \boldsymbol{j}}=$ efecto aleatorio de la interacción entre el $i$ ésimo estado fenológico y el $j$ ésimo animal fistulado;

$\boldsymbol{e}_{\boldsymbol{i j k}}=$ término del error aleatorio asociado a las observaciones, donde $e_{i j k} \sim \operatorname{NIID}\left(0, \sigma^{2}\right)$.

Cuando se observó efecto significativo $(P<0.05)$ del estado fenológico, la prueba de diferencia mínima significativa (LSD), se utilizó para la comparación $(\alpha=0.05)$ de medias.

Los rendimientos de materia seca fueron diferentes $(P<0.05)$ entre etapas (Figura 2). En la etapa de latencia se presentó la mayor producción $\left(2,119 \mathrm{~kg} \mathrm{MS} \mathrm{ha}^{-1}\right)$, debido tal vez a la humedad residual de las precipitaciones ocurridas durante el mes de noviembre. Además, en la Figura 3 se presenta la información del rendimiento de forraje de $M$. repens, ya que fue la especie que presentó rangos desde 72.38 hasta $88.55 \%$ en la composición botánica del área. Sin embargo, en otra investigación ${ }^{(27)}$ reportan producciones de materia seca de $2,913 \mathrm{~kg}$ $\mathrm{ha}^{-1}$ en pastizales invadidos por M. repens en un año lluvioso en Aguascalientes, México; no obstante, durante años secos se obtuvieron producciones de 1,488 $\mathrm{kg} \mathrm{ha}^{-1}$. Lo anterior demuestra que la cantidad de precipitación y humedad en el suelo son factores importantes a considerar. Durante el año de estudio se presentó una precipitación promedio en el área de 
estudio de $510 \mathrm{~mm}$ (considerada atípica), cuando el promedio histórico anual para estas áreas es de $462.4 \mathrm{~mm}^{(14)}$.

Figura 2: Medias de cuadrados mínimos para rendimiento total y rendimiento del Melinis repens $\left(\mathrm{kg} \mathrm{MS} \mathrm{ha}^{-1}\right)$, durante las etapas fenológicas

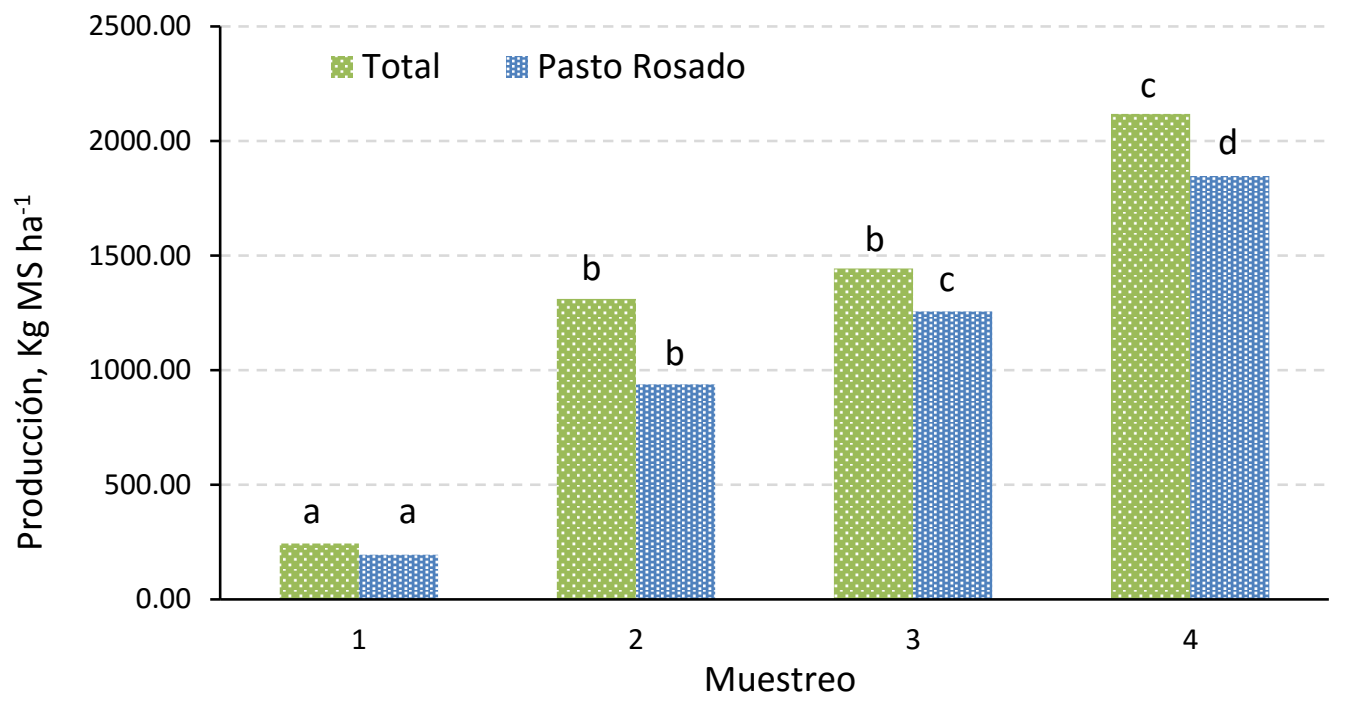

${ }^{\text {abcd }}$ Literales diferentes entre columnas indican diferencia estadística $(P<0.05)$.

En el Cuadro 3 se muestran las especies consumidas por el ganado en pastoreo en las diferentes etapas de muestreo; durante la etapa de latencia $M$. repens fue la especie de mayor contenido en la dieta (35.5\%), comparado con las etapas de crecimiento, madurez y floración $(27,29$ y $29 \%$, respectivamente). El contenido de esta gramínea invasora en la dieta mostró diferencia $(P<0.05)$; ello pudo deberse a que se presentó rebrote durante la época de latencia, el cual fue consumido por el ganado. Además, otros estudios reportan que la abundancia de una especie en un área determinada, puede tener influencia en la dieta de animales en pastoreo $^{(28,29)}$. La dieta consumida por el ganado bovino pastoreando áreas invadidas con M. repens, estuvo constituida principalmente por gramíneas $(74.05 \pm 1.66 \%)$, durante las cuatro etapas fenológicas. Estos resultados son congruentes con lo reportado por otros autores ${ }^{(30)}$, quienes señalaron que las gramíneas constituyen el alimento principal del ganado bovino en pastoreo. 
Cuadro 3: Composición botánica (\%) de la dieta de ganado bovino en un área invadida con Melinis repens

\begin{tabular}{lcccc}
\hline \multirow{2}{*}{ Especie } & \multicolumn{4}{c}{ Etapas de muestreo } \\
\cline { 2 - 5 } Gramíneas & Crecimiento & Floración & Madurez & Latencia $^{\text {Aristida divaricata }}$ \\
Bothriochloa barbinodis & $4.81^{\mathrm{a}}$ & $5.01^{\mathrm{a}}$ & $4.63^{\mathrm{a}}$ & $3.17^{\mathrm{a}}$ \\
Bouteloua chondrosioides & $0.74^{\mathrm{a}}$ & $3.29^{\mathrm{b}}$ & $3.39^{\mathrm{b}}$ & $3.63^{\mathrm{b}}$ \\
Bouteloua curtipendula & $4.06^{\mathrm{a}}$ & $4.96^{\mathrm{a}}$ & $6.02^{\mathrm{a}}$ & $4.97^{\mathrm{a}}$ \\
Bouteloua gracilis & $8.84^{\mathrm{a}}$ & $10.14^{\mathrm{a}}$ & $11.19^{\mathrm{a}}$ & $11.12^{\mathrm{a}}$ \\
Bouteloua hirsuta & $9.23^{\mathrm{a}}$ & $13.59^{\mathrm{b}}$ & $12.07^{\mathrm{ab}}$ & $10.87^{\mathrm{ab}}$ \\
Heteropogon contortus & $2.38^{\mathrm{a}}$ & $4.24^{\mathrm{b}}$ & $3.85^{\mathrm{b}}$ & $4.08^{\mathrm{b}}$ \\
Leptochloa dubia & $0.36^{\mathrm{a}}$ & $2.86^{\mathrm{b}}$ & $3.01^{\mathrm{b}}$ & $2.37^{\mathrm{b}}$ \\
Muhlenbergia phleoides & $0.36^{\mathrm{a}}$ & $3.72^{\mathrm{b}}$ & $2.58^{\mathrm{b}}$ & $2.71^{\mathrm{b}}$ \\
Melinis repens & $0.71^{\mathrm{a}}$ & $2.87^{\mathrm{a}}$ & $1.77^{\mathrm{a}}$ & $2.29^{\mathrm{a}}$ \\
Total & $26.67^{\mathrm{a}}$ & $29.27^{\mathrm{a}}$ & $28.86^{\mathrm{a}}$ & $35.53^{\mathrm{b}}$ \\
Herbáceas & $58.16^{\mathrm{a}}$ & $79.95^{\mathrm{b}}$ & $77.37^{\mathrm{b}}$ & $80.74^{\mathrm{b}}$ \\
Croton pottsii & & & & \\
Total & $12.95^{\mathrm{a}}$ & $5.54^{\mathrm{b}}$ & $4.72^{\mathrm{b}}$ & $4.19^{\mathrm{b}}$ \\
Arbustivas & 12.95 & 5.54 & 4.72 & 4.19 \\
Calliandra eriophylla & & & & \\
Prosopis glandulosa & $15.85^{\mathrm{a}}$ & $8.58^{\mathrm{b}}$ & $11.08^{\mathrm{ab}}$ & $8.95^{\mathrm{b}}$ \\
Total & $13.03^{\mathrm{a}}$ & $5.93^{\mathrm{b}}$ & $6.83^{\mathrm{b}}$ & $6.13^{\mathrm{b}}$ \\
\hline
\end{tabular}

${ }^{\text {ab }}$ Medias con diferente literal en hileras indican diferencia estadística $(P<0.05)$.

Melinis repens fue la especie que presentó el menor índice de preferencia (desde 0.33 hasta 0.41) durante las cuatro etapas, a pesar de su alta densidad en el área de estudio. Sin embargo, Bouteloua chondrosioides presentó un índice alto (16.26) durante la madurez, mientras que Aristida divaricata presentó un índice de 8.43 durante la etapa de crecimiento (Cuadro 4). A pesar de la baja densidad de estas especies en el área de estudio, presentaron los índices de preferencia más altos. Este comportamiento es respaldado por estudios previos ${ }^{(28,31)}$, donde se observa que las especies que presentaron mayor índice de preferencia, fueron las de menor presencia en el área de estudio. 
Cuadro 4: Índice de preferencia en un área invadida por Melinis repens

\begin{tabular}{|c|c|c|c|c|}
\hline \multirow{2}{*}{ Especie } & \multicolumn{4}{|c|}{ Etapas de muestreo } \\
\hline & Crecimiento & Floración & Madurez & Latencia \\
\hline \multicolumn{5}{|l|}{ Gramíneas } \\
\hline Aristida divaricata & $8.43^{1 \mathrm{a}}$ & $--^{2}$ & -- & $5.19^{\mathrm{a}}$ \\
\hline Bothriochloa barbinodis & -- & $0.80^{\mathrm{a}}$ & $2.95^{\mathrm{ab}}$ & $4.49^{\mathrm{b}}$ \\
\hline Bouteloua chondrosioides & -- & $1.06^{\mathrm{a}}$ & $16.26^{\mathrm{b}}$ & $5.02^{\mathrm{a}}$ \\
\hline Bouteloua curtipendula & $0.92^{\mathrm{a}}$ & $1.33^{\mathrm{a}}$ & $3.43^{\mathrm{b}}$ & $3.09^{\mathrm{b}}$ \\
\hline Bouteloua gracilis & $0.91^{\mathrm{a}}$ & $1.85^{\mathrm{b}}$ & $2.47^{\mathrm{bc}}$ & $3.11^{\mathrm{c}}$ \\
\hline Bouteloua hirsuta & -- & $1.74^{\mathrm{a}}$ & $2.20^{\mathrm{ab}}$ & $3.09^{\mathrm{b}}$ \\
\hline Heteropogon contortus & -- & -- & $2.98^{\mathrm{a}}$ & $1.60^{\mathrm{a}}$ \\
\hline Leptochloa dubia & -- & $3.36^{\mathrm{a}}$ & -- & $3.01^{\mathrm{a}}$ \\
\hline Muhlenbergia phleoides & -- & $2.84^{\mathrm{a}}$ & $2.91^{\mathrm{a}}$ & $2.66^{\mathrm{a}}$ \\
\hline Melinis repens & $0.34^{\mathrm{a}}$ & $0.41^{\mathrm{a}}$ & $0.33^{\mathrm{a}}$ & $0.41^{\mathrm{a}}$ \\
\hline \multicolumn{5}{|l|}{ Herbáceas } \\
\hline Croton pottsii & $12.95^{\mathrm{a}}$ & $5.54^{\mathrm{b}}$ & $4.72^{\mathrm{b}}$ & $4.19^{\mathrm{b}}$ \\
\hline \multicolumn{5}{|l|}{ Arbustivas } \\
\hline Calliandra eriophylla & $1.65^{\mathrm{a}}$ & $0.89^{\mathrm{b}}$ & $1.15^{\mathrm{ab}}$ & $0.93^{\mathrm{ab}}$ \\
\hline Prosopis glandulosa & $5.21^{\mathrm{a}}$ & $2.37^{\mathrm{b}}$ & $2.73^{\mathrm{b}}$ & $2.45^{\mathrm{b}}$ \\
\hline
\end{tabular}

El contenido de proteína cruda $(\mathrm{PC})$ fue diferente $(P<0.05)$ entre etapas fenológicas. Los mayores valores se encontraron durante la etapa de crecimiento y floración (13 y $11 \%$, respectivamente). En contraste, los valores más bajos se encontraron durante la etapa de latencia (6.5\%; Cuadro 5). Sin embargo, en otro estudio reportan valores de PC de $10.49 \%$ durante el verano y $5.49 \%$ durante la primavera; por lo que se puede atribuir que los animales no cubren sus requerimientos nutricionales ${ }^{(32)}$. Además, otros autores reportan que el ganado bovino durante la época de lluvias, puede cubrir sus requerimientos mínimos de mantenimiento en el norte de México, esto debido a la fenología que presentan los pastos ${ }^{(33)}$; sin embargo, durante el invierno los nutrientes disminuyen, lo cual provoca que no cumplan sus requerimientos durante esta época ${ }^{(34)}$. Los valores de PC registrados en la presente investigación durante la etapa de crecimiento y floración, se debieron probablemente al consumo de especies con valores altos de PC, tales como Bouteloua gracilis y Bouteloua curtipendula; arbustivas como Prosopis glandulosa y Calliandra eriophylla y la herbácea Croton pottsii ${ }^{(35,36,37)}$. Además, la temporada es otro factor a considerar en estudios de esta 
naturaleza ${ }^{(38)}$. Por lo anterior, se puede asumir que los valores reportados de PC no se debieron al consumo de Melinis repens, ya que esta especie presenta valores de PC desde 4 hasta $6 \%^{(7)}$.

Cuadro 5: Medias $( \pm \mathrm{EE})$ de la composición química de la dieta consumida por bovino en pastoreo en un área invadida por Melinis repens, durante las etapas fenológicas

\begin{tabular}{lcccc}
\hline $\begin{array}{l}\text { Variable } \\
(\boldsymbol{\%})\end{array}$ & Crecimiento & Floración & Madurez & Latencia \\
\cline { 2 - 5 } & $13.76 \pm 0.92^{\mathrm{a}}$ & $10.72 \pm 0.92^{\mathrm{b}}$ & $8.61 \pm 0.92^{\mathrm{bc}}$ & $6.559 \pm 0.92^{\mathrm{c}}$ \\
PC & $85.86 \pm 0.607^{\mathrm{a}}$ & $85.95 \pm 0.607^{\mathrm{a}}$ & $81.39 \pm 0.607^{\mathrm{b}}$ & $81.10 \pm 0.607^{\mathrm{b}}$ \\
$\mathrm{MO}$ & $41.36 \pm 1.86^{\mathrm{a}}$ & $38.53 \pm 1.86^{\mathrm{a}}$ & $43.08 \pm 1.86^{\mathrm{a}}$ & $36.56 \pm 1.86^{\mathrm{a}}$ \\
D $i v \mathrm{MO}$ & $70.71 \pm 1.54^{\mathrm{a}}$ & $71.19 \pm 1.54^{\mathrm{a}}$ & $71.02 \pm 1.54^{\mathrm{a}}$ & $72.64 \pm 1.54^{\mathrm{a}}$ \\
FDN & $42.80 \pm 1.38^{\mathrm{a}}$ & $42.46 \pm 1.38^{\mathrm{a}}$ & $47.74 \pm 1.38^{\mathrm{a}}$ & $42.70 \pm 1.38^{\mathrm{a}}$ \\
\hline FDA &
\end{tabular}

$\mathrm{PC}=$ proteína cruda; $\mathrm{MO}=$ materia orgánica; $\mathrm{D} i v \mathrm{MO}=$ digestibilidad in vitro de la materia orgánica.

FDN: FDA= fibra detergente neutro y ácido.

${ }^{\text {abc }}$ Medias con diferente literal en hileras indican diferencia $(P<0.05)$.

La DivMO fluctuó entre 36.56 y $43.08 \%$ ( $P>0.05$; Cuadro 5). Lo anterior se puede deber al cambio estructural de las gramíneas, hierbas y arbustivas durante el desarrollo de las plantas, lo cual afecta la cantidad de tejidos digestibles ${ }^{(39)}$. Otros autores han reportado valores desde 52.3 hasta $54.9 \%$ en áreas invadidas por Melinis repens durante la época de latencia ${ }^{(13)}$. Este resultado concuerda con lo reportado en otro trabajo, donde se reportan valores de DivMO de 67.34 y $58.23 \%$ en verano y primavera, respectivamente ${ }^{(32)}$.

En el Cuadro 5 se muestran los datos obtenidos para fibra detergente neutro (FDN), donde no se encontraron diferencias $(P>0.05)$; sin embargo, fueron diferentes a los obtenidos en otro estudio $^{(32)}$, donde se reportaron valores desde 64 hasta $74 \%$ de FDN en un pastizal con presencia de Melinis repens. También, otros investigadores ${ }^{(40,41)}$ reportaron valores desde 69.2 hasta $70.1 \%$, similares a los obtenidos en esta investigación. Además, Murillo ${ }^{(42)}$ reporta valores de FDN (70.4\%) similares a los obtenidos en este estudio, durante la época de latencia. De la misma manera se obtuvo la FDA, donde no se encontraron diferencias $(P>0.05)$. Sin embargo, otros autores reportan valores mayores a los obtenidos en este estudio $^{(32)}$. A mayor madurez el contenido de polisacáridos lignificados se incrementa y por tanto el valor de la FDA también se incrementa ${ }^{(43)}$. Debido a lo anterior, probablemente los valores de FDA en la dieta obtenidos en la presente investigación, oscilaron desde 42.7 hasta $47.74 \%$. Un estudio reciente ${ }^{(41)}$ reporta valores de FDA (42.3\%) similares a los obtenidos en este estudio. 
La composición botánica de la dieta estuvo constituida principalmente por Melinis repens durante las cuatro etapas fenológicas, ya que ésta fue la gramínea más abundante en el área de estudio. Las gramíneas fueron preferidas por los animales durante las cuatro fechas de muestreo y sólo se presentó una herbácea (Croton pottsii) durante el muestreo. Los mayores índices de preferencia para $M$. repens se obtuvieron durante las etapas de latencia y floración. El valor nutricional de la dieta de bovinos en pastoreo en áreas invadidas por Melinis repens, no satisface las necesidades requeridas para producción y mantenimiento durante las fechas de madurez y latencia. Es conveniente realizar estudios enfocados a la planeación y diseño de esquemas de utilización en pastizales invadidos con especies exóticas.

\section{Agradecimientos}

Al Consejo Nacional de Ciencia y Tecnología (CONACyT), a la Universidad Autónoma de Chihuahua (UACH) y a la Fundación Produce Chihuahua por su apoyo financiero. Además, al Colegio de Posgraduados por permitir el uso de las instalaciones para la realización del trabajo de investigación.

\section{Literatura citada:}

1. INEGI. Instituto Nacional de Estadística y Geografía. Anuario estadístico y geográfico de Chihuahua. Ed. Instituto Nacional de Estadística y Geografía. Aguascalientes, México. 2015.

2. INEGI. Instituto Nacional de Estadística y Geografía. Anuario estadístico del Estado de Chihuahua. Gobierno de Chihuahua. 1995.

3. Báez GAD, Reyes LG, Melgoza CA, Royo MM, Carrillo RR. Características productivas del sistema vaca-cría en el estado de Chihuahua. Tec Pecu Mex 1999;37(2):11-24.

4. SNIIM. Sistema nacional de información e integración de mercados. Mercados del exterior. En: http://www. economia-sniim.gob.mx/nuevo. Consultado 17 Oct 2013.

5. Callejas JN, Aranda GH, Rebollar RS, De la Fuente MM. Situación económica de la producción de bovinos de carne en el Estado de Chihuahua, México. Agron Mesoam 2014;25(1):133-139. 
6. PACP-Ch. Plan de acción para la conservación y uso sustentable de los pastizales del desierto chihuahuense en el Estado de Chihuahua 2011-2016, Guzmán-Aranda JC, et al editores. Gobierno del Estado de Chihuahua, México. 2011.

7. Melgoza CA, Balandrán MI, Mata GR, Pinedo C. Biología del pasto rosado Melinis repens (Will.) e implicaciones para su aprovechamiento o control. Revisión. Rev Mex Cienc Pecu 2014;5(4):429-442.

8. Villaseñor RJL, Espinosa GFJ. Catálogo de malezas de México. Universidad Nacional Autónoma de México. Consejo nacional consultivo fitosanitario. Fondo de Cultura Económica. México, DF. 1998.

9. Chávez SAH, González MH, Fierro LC. Consumo voluntario de forraje en vacas gestantes durante la época de sequía. Bol Pastizales. RELC-INIP-SARH. 1981.

10. Chávez SAH, Fierro LC, Habib R, Sánchez E, Ortiz V. Composición botánica y valor nutricional de la dieta de bovinos en un pastizal mediano abierto en la región central de Chihuahua. Téc Pecu Méx 1986;50:90-105.

11. Peña JM, Habib R. La técnica microhistológica; un método para determinar la composición botánica de la dieta de herbívoros. INIP-SARH. 1980;1:3-82.

12. Holechek JL, Vavra M, Pieper RD. Methods for determining the nutritive quality of range ruminant diets: A review. J Anim Sci 1982:54(2):363-376.

13. González FJ, Martínez M, Serna O, Carrete F. Georreferenciación de la dieta del ganado bovino durante el período de sequía en diferentes localidades en Durango. RELCINIFAP. 2012.

14. Medina G, Díaz G, Berzoza M, Silva MM, Chávez AH, Báez AD. Estadísticas climatológicas básicas del Estado de Chihuahua (periodo 1961-2003). INIFAP-CIRNC. Libro técnico No 1. 2006.

15. CONAGUA. Reporte del clima en México. Coordinación general del servicio meteorológico nacional gerencia de meteorología y climatología subgerencia de pronóstico a mediano y largo plazo. Reporte Anual 2013.

16. COTECOCA. Comisión Técnica Consultiva para la Determinación de los Coeficientes de Agostadero. Determinación de los Coeficientes de Agostadero Chihuahua. Secretaria de Agricultura y Recursos Hidráulicos. México. 1978.

17. Chávez SAH, Pérez GA, Sánchez GE. Intensidad de pastoreo y esquema de utilización en la selección de la dieta del ganado bovino durante la sequía. Téc Pecu Méx 2000;38(1):19-34. 
18. Herrick JE, Van Zee JW, Havstad KM, Burkett LM, Whitford WG. Monitoring manual for grassland, shrubland and savanna ecosystems. Volume I: Quick start. USDA - ARS Jornada Experimental Range. Las Cruces, New Mexico. 2009.

19. Holechek JL, Vavra M. The effects of slide and frequency observation numbers on the precision of microhistological analysis. J Range Manage 1981;34(4):337-338.

20. Holechek JL, Vavra M. Fistula sample numbers required to determine cattle diets on forest and grassland ranges. J Range Manage 1983;36:323-326.

21. Ellis WC, Bailey EM, Taylor CA. A silicone esophageal cannula; its surgical installation and use in research with grazing cattle, sheep or goats. J Anim Sci 1984;59(1):204-209.

22. AOAC. Official methods of analysis of the assoc. off anal. chem. 19a ed. Wahington, DC. USA. 2012.

23. Ankom Technology. Procedures for fiber and in vitro analysis. https://www.ankom.com/product-catalog/daisy-incubator. Consultada 16 Nov, 2014.

24. Van Dyne GM, Heady HF. Botanical composition of sheep and cattle diets on a mature annual range. Hilgardia 1965;36:465-492.

25. Van Soest, PJ, Robertson JB, Lewis BA. Methods for dietary fiber, neutral detergent fiber, and non-starch polysaccharides in relation to animal nutrition. J Dairy Sci 1991;74(10):3583-3597.

26. SAS Institute Inc. SAS 9.1.3 User's guide. Cary, NC, USA. 2006.

27. Díaz RA, Flores E, De Luna A, Luna JJ, Frías JT, Olalde V. Biomasa aérea, cantidad y calidad de semilla de Melinis repens (Willd.) Zizka, en Aguascalientes, México. Rev Mex Cienc Pecu 2012;3(1):33-47.

28. Launchbaugh KL, Stuth JW, Holloway JW. Influence of range site on diet selection and nutrient intake of cattle. J Range Manage 1990;43:109-115.

29. Ralphs MH, Pfister JA. Cattle diets in tall forb communities on mountain rangelands. J Range Manage 1992;45:534-537.

30. Beck JL, Peek JM. Diet composition, forage selection, and potential for forage competition among elk, deer, and livestock on aspen-sagebrush summer range. Rangeland Ecol Manag 2005;58(2):135-147.

31. Vásquez F, Pezo D, Mora DJ, Skarpe C. Selectividad de especies forrajeras por bovinos en pastizales seminaturales del trópico centroamericano: un estudio basado en la observación sistemática del pastoreo. Zootecnia Trop 2012;30: 063-080. 
32. Reyes-Estrada O, Murillo-Ortiz M, Herrera-Torres E, Gurrola-Reyes JN, CarreteCarreón FO. Cambios estacionales en consumo, composición química y degradabilidad ruminal de la dieta seleccionada por novillos en pastoreo. Ecosistemas y Recursos Agropecuarios 2014;1(2):97-106.

33. Murillo OM, Reyes EO, Herrera TE, Martínez GJH, Villareal RG. Annual and seasonal variation in nutritive quality and ruminal fermentation patters of diets in steers grazing native rangelands. African J Agr Res 2013;8(33):4408-4413.

34. Villalobos GC, González VE, Ortega SJA. Técnicas para estimar la degradación de la proteína y materia orgánica en el rumen y su importancia en rumiantes en pastoreo. Téc Pecu Méx 2000;38(2):119-134.

35. Tena J, Ortiz V, Gómez F. Composición química de zacates nativos, introducidos y arbustivas en cuatro estados fenológicos. Bol. Pastizales. RELC-INIP-SARH. 1984.

36. CIPES. Centro de Investigaciones Pecuarias del Estado de Sonora. Manejo de Pastizales. 20 años de investigación pecuaria en el estado de Sonora. INIFAP-SARH. Gobierno del Estado. UGRS. 1989.

37. Morales NC, Madrid L, Melgoza A, Martínez M, Arévalo S, Rascón Q, Jurado P. Análisis morfológico de la diversidad del pasto navajita [Bouteloua gracilis (Willd. ex Kunth) Lag. ex Steud.], en Chihuahua, México. Téc Pecu Méx 2009;47(3):245-256.

38. Reyes O, Murillo M, Herrera E, Carrete FO. Seasonal and annual changes in the quality of native rangeland selected by grazing steers in northern Mexico. Cienc Inv Agr 2016;43(2):203-212

39. Vázquez de Aldana BR, García CA, Pérez CE, García CB. Elemental content in grassland of semiarid zones: effect of topographic position and botanical composition. Commun Soil Sci Plant Anal 1993;24(15-16):1975-1989.

40. Olson KC. Diet sample collection by esophageal fistula and rumen evacuation techniques. J Range Manage 1991;44:515-519.

41. Clark A Del Curto T, Vavra M, Dick BL. Stocking rate and fuels reduction effects on beef cattle diet composition and quality. Rangeland Ecol Manag 2013;66:714-720.

42. Murillo OM, Palacio CC, Reyes EP, Carrete CFO, Ruiz BO. Valor nutricional de la dieta consumida por ganado bovino en un pastizal mediano arbosufrutescente durante tres estaciones del año. AGROFAZ 2004;4:561-566.

43. Scales, CH, Streeter CL, Denham AH, Ward GM. Effect of mastication, salivary contamination and leaching on the chemical composition of forage samples collected via esophageal fistulae. J Anim Sci 1974;38(6):1278-1283. 